the number of particles emitted. This is not possible in a sensible renormalisable theory which should give finite results at all energies and for any number of particles. In renormalisable theories all processes to any order in the coupling constant are calculable in principle.

Salam ${ }^{11}$ in particular emphasised the importance of the renormalisability criterion and thought that it should follow as in QED from a principle of gauge invariance. In QED this is the statement that the components of the electromagnetic field vector $A_{\lambda}$ in the direction of the photon momentum $k_{\lambda}$ are redundant. It follows just as in classical electrodynamics that the photon (or electromagnetic wave) can only possess transverse modes, thereby removing the difficulty inherent in longitudinal modes. However, for QED to be gauge invariant the photon must have zero mass. Yang-Mills theory involves a more complicated gauge invariance where the 'photons' must also have zero mass.

The problem of inducing a vector boson mass without destroying the gauge invariance of the theory was actually solved in 1964 in some seemingly obscure work based on the theory of superconductivity. In superconductivity the ground state of the system is not invariant under the full symmetry of the interaction describing the system (a simpler example is ferromagnetism where the direction of the ferromagnet in its ground state clearly breaks the rotational symmetry of the underlying spin-spin interaction); an energy gap thus appears between the ground state and the lowest symmetric state. Nambu and JonaLasinio $^{12}$ showed that this process of spontaneous symmetry breaking (SSB) can be considered as the generation of mass for a fermion initially with zero mass but with a pairing interaction as in superconductivity. The energy gap behaves like a mass.

Brout and Englert ${ }^{13}$ and Higgs ${ }^{14}$ then showed that if one starts with a gauge theory involving a zero mass vector boson interacting with scalar fields, then SSB will generate a mass for the vector boson without destroying the gauge invariance of the underlying interaction. So it is possible that the good (that is, renormalisable) properties of the underlying theory are retained. In this case at least one scalar particle must also exist as a result of SSB.

It is surprising in retrospect that it took physicists three years to incorporate the ideas of SSB into unified weak-electromagnetic theory. In 1967 Weinberg ${ }^{15}$ applied these ideas to Glashow's $S U(2) \times U(1)$ theory thereby predicting the mass relation $M_{W}=M_{Z} \cos \theta$ where $\theta$ is Glashow's mixing angle so giving the strength $\mathrm{G}^{\prime}$ of leptonic neutral currents as $\mathrm{G}^{\prime}=\frac{1}{2} \mathrm{G}_{\mu}$. Furthermore, the mass $\mathrm{M}_{\mathrm{W}}$ is $37 /(\sin \theta) \mathrm{GeV}$ or about $78 \mathrm{GeV}$ with the present value for $\sin \theta$. Salam at about the same time was applying the ideas of SSB to his own and Ward's version of the theory. His results ${ }^{16}$ are in agreement with those of Weinberg but his more general approach

\title{
What the theory is about
}

EINSTEIN spent the latter half of his life struggling to find a unified theory of the gravitational force and electromagnetism; he failed because he could not transfer the geometrical ideas of the general theory of relativity (his spacetime theory - still the current theory of gravitation) to the electromagnetic field. However he twisted and turned his electromagnetic 'vectors' and 'tensors' electromagnetism remained stubbornly non-geometrical: the electric and magnetic forces were something you simply had to add to space-time, like actors on a stage.

But now Glashow, Salam, and Weinberg, and the physicists who worked before and with them (see accompanying article) have turned the tables on Einstein by unifying electromagnetism with something else - the weak interaction, which if is responsible for the 'beta decay' of radioactive nuclei and for the transformation of protons into neutrons (and hence hydrogen into helium) that allows the Sun to burn. Both electromagnetism and the weak force are described in the theory as fields of force laid upon space-time; but they are now conceived as aspects of the same field of force, seen in different circumstances, just as magnetism and lightning are different aspects of the electromagnetic field.

Electricity and magnetism, incidentally, were unified by the Scot James Clerk Maxwell towards the end of the 19th century, in a 'field theory' which is still very much at the root of modern theories of fields of force. Maxwell showed that there must be propagating waves of electromagnetic field, and that they would travel at the speed of light; Hertz demonstrated their existence.

Light is an electromagnetic wave radiated by moving electric charges. It follows that if the weak force is to be combined in a unified theory with electromagnetism, there must be 'weak' charges and a corresponding radiation. In particle language (corresponding to the invocation of quantum mechanics, and the 'quantisation' of the field) there are photons (for light) and what came to be known as 'intermediate vector bosons' for the weak radiation. The difference is that photons are massless, and can cause interactions at infinitely long range, whereas the IVBs are massive and cause

did not allow him to derive explicit mass relations for $\mathrm{M}_{\mathrm{W}}$ and $\mathrm{M}_{\mathrm{Z}}$.

The rest is history. On the theoretical side, Glashow, Illiopoulos and Maiani ${ }^{17}$ in 1970 introduced the concept of charm (Nature 262, 537; 1976) thereby allowing the unified theory to be applied to hadrons and not only to leptons; the following year 't Hooft ${ }^{18}$ showed in a tour de force that the Weinberg model was renormalisable. short-range forces. Thus the weak field of protons and neutrons cannot be felt outside the atomic nucleus whereas their electric field extends outwards and captures electrons, thus creating atoms. Inside the nucleus, the weak field causes beta dacay.

So far so good, but we still only have parallel theories, not unified ones. The next step was highly technical, and took a long time to emerge, but has very interesting consequences. It proved that the weak and electromagnetic fields could be understood as aspects of the same field only if the 'uni-field' was being propagated though a physical medium, the Higgs medium spreading throughout space-time. The medium was rather like calcite, which splits light into two beams according to its polarisation; the Higgs medium splits the uni-field (which has massless, long-range particles) into a heavy part (the IVBs) and a massless remainder (light).

Back to the aether, do I hear? Not quite. The medium is supposed to be real and detectable, and it obeys all the constraints of special relativity. Since it is uniform throughout space-time it is not noticeable until it is altered locally in some way, which cannot be done except at very high particle energies; but at those energies it is possible to predict that 'Higgs particles' will be created, like splashes in the medium (they will correspond roughly to density waves like sound in air).

The theory also predicts the exact masses and charges of the IVBs (there will be three, positive, negative, and neutral); and although it has been well verified at low energies in experiments with electrons and neutrinos, the real test will come with the detection (or not!) of the IVBs and the Higgs particles. Meanwhile particle theorists are attempting to apply the techniques of Higgs media (or 'spontaneous symmetry breaking' as it is called) to a unification of the weak, electromagnetic, and strong forces without, yet, it must be admitted, much success.

Once the experiments that test the Glashow-Salam-Weinberg model have been done we shall know whether we are on the right lines or whether we have to start along a completely new track.

Robert Walgate

Experimentally, weak neutral currents were discovered in the remarkable experiment ${ }^{20}$ at SLAC last year (Nature 274, 11; 1978), when it was shown that left-handed electrons scatter off deuterons more than right-handed electrons.

In conclusion it is only fair to give credit to those whose work was inextricably linked with the development of the theory, which as is clear was very much a 